\title{
Ampere per Meter
}

National Cancer Institute

\section{Source}

National Cancer Institute. Ampere per Meter. NCI Thesaurus. Code C68830.

A SI derived unit used for linear electric current density, magnetic field strength, and magnetization. It is the magnetic auxiliary field strength created tang entially at a distance of one meter from a straight conductor of infinite length and with neg ligible cross section by a circulating current of one ampere, or linear electric current density when one ampere electric current is conducted in one meter wide conducting sheet. 